\title{
Protecting the very young against pertussis - cough, costs and cocooning
}

D Lévy-Bruhl (d.levybruhl@invs.sante.fr) ${ }^{1}$

1. Institut de veille sanitaire (InVS), Saint Maurice, France

Citation style for this article:

Lévy-Bruhl D. Protecting the very young against pertussis - cough, costs and cocooning. Euro Surveill. 2014;19(5):pii=20689. Available online: http://www. eurosurveillance.org/ViewArticle.aspx?Articleld $=20689$

The conclusions of the paper by Lim et al., in this issue of Eurosurveillance, assessing the relevance of a pertussis cocoon immunisation strategy in Ontario, are somewhat disappointing [1]. The authors estimate the number of persons that need to be vaccinated (NNV) in order to prevent an infant pertussis hospitalisation or death at 12,000 to 63,000 and 1.1 million to 12.8 million, respectively and conclude to the 'inefficiency inherent to this approach'. These high NNV seem little debatable - the analysis is well conducted. Furthermore, the authors have explored in their sensitivity analysis various assumptions and values that could have led them to overestimate the NNV in the base-case analysis. Their conclusions are robust and in line with a similar study conducted in Quebec and British Columbia for the years 2005 to 2009 , which also concluded that in the context of low pertussis incidence, the parental cocoon strategy is inefficient and resource intensive' [2].

The idea behind the cocoon strategy is attractive in principle. Available pertussis vaccines and vaccination strategies do not allow the elimination of pertussis. Even a combination of very high vaccination coverage in infants, with additional boosters later in life, does not induce sufficient herd immunity to prevent the circulation of the bacteria in adults. This leaves us with a persisting burden of the disease and the resulting mortality is almost exclusively concentrated in the first weeks or months of life. This is due to a combination of two factors. Firstly, even though pertussis can be severe at all ages, it is most severe in infants and may lead to pulmonary and neurological complications and death. Secondly, newborns remain susceptible to pertussis until they receive at least the first dose of a pertussis-containing vaccine, which in all European Union Member States is given at two or three months of age. In the absence of pertussis vaccines for use in the neonatal period, young infants can thus only be indirectly protected. This can be achieved through vaccinating those who are most likely to contaminate them. This seems to make sense, given that very young infants have limited social contacts and the potential main contaminators are probably not too numerous and easy to identify. Indeed, countries having embarked on such a strategy mainly target household members for vaccination and such choice is supported by data. A recent review has concluded that, when the source of pertussis transmission to infants less than six months old is identified, parents contribute with between $39 \%$ and $57 \%$ and siblings with between $16 \%$ and $42 \%$ [3].

In this context, what conclusions can be inferred from Lim's results? The answer is not straightforward and needs further consideration.

Lim et al. cautiously point out that their conclusions stand for Ontario, which has a very low burden of severe pertussis in infants, and may not be relevant to contexts with different pertussis epidemiology. In addition to this, one needs to keep in mind the cyclical pattern of pertussis epidemiology. What is true for a period of low incidence may not apply during periods of resurgence of the disease. In France, 10 infant deaths were registered in 2000 and 2005 , at the peak of epidemic cycles, and in the United Kingdom, 14 infant deaths were registered during the 2012 pertussis resurgence, making protection of young infants worth considering [4-5].

In their paper, Lim et al. measure the relevance of the immunisation strategy through the NNV. This metric represents the ratio of newly vaccinated individuals to cases prevented. It is defined as the reciprocal of the product of the vaccine effectiveness by the attack rate in the unvaccinated. Putting aside the imperfect effectiveness of a vaccine, the NNV is a reflection of the risk of the disease in the absence of vaccination. While this approach is definitively very informative, it only reflects the epidemiological benefit of the vaccination. Vaccination decisions usually also consider the riskbenefit balance and the cost-effectiveness ratio. If a vaccine is perfectly tolerated, with no detrimental indirect effect, the risk-benefit balance may be in favour of vaccination, even with a high NNV. In adults primed with pertussis whole-cell vaccines, the addition of an acellular pertussis component does not modify the safety profile of the Tetanus-Diphtheria (Td) boosters, at least for the first doses [6]. If a vaccine is cheap and adds only little to the costs of a programme, the costeffectiveness ratio may again be favourable even with 
a high NNV. Two Dutch studies have looked at the costeffectiveness ratio of a pertussis cocooning strategy [7-8]. They come to conflicting conclusions in the basecase analysis with cost-effectiveness ratios of 4,600 and 89,000 EUR, respectively, per quality-adjusted life year (QALY) gained as compared to the Dutch (unofficial) cost-effectiveness threshold of 20,000 to 50,000 EUR/QALY gained [7]. The differences mainly reflect diverse assumptions regarding the level of underreporting and duration of the disease. However, in the sensitivity analysis of the least favourable study, the combination of the most favourable assumptions of the variables leads to a cost-effectiveness ratio of only 21,000 EUR/QALY gained. In contrast to these positive results, an Italian study has, similarly to Lim et al., concluded that cocooning would be poorly efficient, based on costs of over 100,000 EUR per hospitalisation avoided [9].

In addition to considering costs, it is important to look at feasibility and acceptability of vaccination strategies. The pertussis cocooning immunisation requires administering unneeded diphtheria and tetanus boosters, as there is no monovalent pertussis vaccine available to date. Moreover, it does not easily fit into existing vaccination practices as it cannot rely on an already implemented vaccination strategy targeting members of a family. One would imagine that the acceptability of household members for a vaccination aiming at preventing risk of a severe disease for the young infant in the happy context of a future or recent birth, should be high. However, such an optimistic view remains to be confirmed in real life. The recommendation of an adult pertussis booster dose that relies on the simple substitution of a pertussis-containing vaccine (Tdap) at the occasion of a planned $\mathrm{Td}$, as implemented in Ontario and elsewhere, appears easier to implement than the cocooning strategy. However, had solely the NNV to prevent a hospitalisation or a death been used to decide on this strategy, it would probably not have been implemented. Hospitalisations and deaths due to pertussis in adults are respectively very rare and almost inexistent. At the same time, such a strategy can only impact on severe cases in infants when the vaccine is given by chance to an adult who is or will be in the next years in regular close contact with an infant. Only if regular boosters were proposed, could a significant impact on infant pertussis be expected through herd immunity. Still, the NNV to prevent a pertussis case in adults is currently challenged by the likely shorter than expected duration of protection conferred by boosters, as suggested by recent data in adolescents [10-11].

There are nevertheless some new approaches that give hope for improving the protection of the very young such as the promising preliminary results of the temporary maternal pertussis immunisation strategy implemented in England since 2012 [12]. Pertussis is the single 'old' vaccine-preventable disease that resists vaccination and even mocks us with its resurgence observed in many places in the recent years, despite high vaccine coverage in children and booster doses added in the immunisation schedules at different ages. Even if adults will probably continue to suffer from exhausting coughs due to pertussis for many more years, we may soon have new vaccination strategies at hand, namely maternal or neonatal vaccinations, to eliminate infant pertussis deaths. These are of course subject to the absence of any safety issue and to high acceptability of the strategy and to the various NNV. New calculations, new debates to come...

\section{References}

1. $\operatorname{Lim} \mathrm{GH}$, Deeks SL, Crowcroft NS. A cocoon immunisation strategy against pertussis for infants: does it make sense for Ontario? . Euro Surveill. 2014;19(5):pii=20688.

2. Skowronski DM, Janjua NZ, Tsafack EP, Ouakki M, Hoang L, De Serres $G$. The number needed to vaccinate to prevent infant pertussis hospitalization and death through parent cocoon immunization. Clin Infect Dis. 2012;54(3):318-27. http://dx.doi.org/10.1093/cid/cir836

3. Wiley KE, Zuo Y, Macartney KK, McIntyre PB. Sources of pertussis infection in young infants: a review of key evidence informing targeting of the cocoon strategy. Vaccine. 2013;31(4):618-25. http://dx.doi.org/10.1016/j. vaccine.2012.11.052

4. Public Health England (PHE). Pertussis notifications and deaths, England and Wales, 1940 - 2012. London: PHE. 6 Dec 2013. Available from: http://www.hpa.org.uk/webc/ HPAwebFile/HPAweb_C/1317133571994

5. Institut national de la santé et de la recherche médicale (Inserm). Centre d'épidémiologie sur les causes médicales de décès (CépDc). Interrogation des données sur les causes de décès de 1979 à 2011. Paris: Inserm/ CépDc. [Accessed 3 Feb 2014]. Available from: http://www.cepidc.inserm.fr/inserm/ $\mathrm{html} /$ index2.htm

6. Pichichero ME, Blatter MM, Kennedy WA, Hedrick J, Descamps $D$, Friedland LR. Acellular pertussis vaccine booster combined with diphtheria and tetanus toxoids for adolescents. Pediatrics. 2006;117(4):1084-93. http://dx.doi.org/10.1542/ peds.2005-1759

7. Lugner AK, van der Maas N, van Boven M, Mooi FR, de Melker $\mathrm{HE}$. Cost-effectiveness of targeted vaccination to protect newborns against pertussis: comparing neonatal, maternal, and cocooning vaccination strategies. Vaccine. 2013;31(46):5392-7. http://dx.doi.org/10.1016/j.vaccine.2013.09.028

8. Westra TA, de Vries R, Tamminga JJ, Sauboin CJ, Postma MJ. Cost-effectiveness analysis of various pertussis vaccination strategies primarily aimed at protecting infants in the Netherlands. Clin Ther. 2010;32(8):1479-95. http://dx.doi.org/10.1016/j.clinthera.2010.07.017

9. Meregaglia M, Ferrara L, Melegaro A, Demicheli V. Parent "cocoon" immunization to prevent pertussis-related hospitalization in infants: the case of Piemonte in Italy. Vaccine. 2013;31(8):1135-7. http://dx.doi.org/10.1016/j.vaccine.2012.12.061

10. Witt MA, Katz PH, Witt DJ. Unexpectedly limited durability of immunity following acellular pertussis vaccination in preadolescents in a North American outbreak. Clin Infect Dis. 2012;54(12):1730-5. http://dx.doi.org/10.1093/cid/cis287

11. Klein NP, Bartlett J, Rowhani-Rahbar A, Fireman B, Baxter R. Waning protection after fifth dose of acellular pertussis vaccine in children. N Engl J Med. 2012;367(11):1012-9. http://dx.doi.org/10.1056/NEJMoa1200850

12. Salisbury D. Renewing the fight against whooping cough. London: Department of Health. 19 Jul 2013. Available from: https://www.gov.uk/government/speeches/ renewing-the-fight-against-whooping-cough 